\title{
Deciphering the archaeal communities in tree rhizosphere of the Qinghai-Tibetan plateau
}

\author{
Mengjun Zhang, Liwei Chai, Muke Huang, Weiqian Jia, Jiabao Guo and Yi Huang*
}

\begin{abstract}
Background: The Qinghai-Tibetan Plateau represents one of the most important component of the terrestrial ecosystem and a particularly vulnerable region, which harbouring complex and diverse microbiota. The knowledge about their underground microorganisms have largely been studied, but the characteristics of rhizosphere microbiota, particularly archaeal communities remains unclear.

Results: High-throughput Illumina sequencing was used to investigate the rhizosphere archaeal communities of two native alpine trees (Picea crassifolia and Populus szechuanica) living on the Qinghai-Tibetan Plateau. The archaeal community structure in rhizospheres significantly differed from that in bulk soil. Thaumarchaeota was the dominant archaeal phylum in all soils tested (92.46-98.01\%), while its relative abundance in rhizospheres were significantly higher than that in bulk soil. Ammonium nitrogen, soil organic matter, available phosphorus and pH were significantly correlated with the archaeal community structure, and the deterministic processes dominated the assembly of archaeal communities across all soils. In addition, the network structures of the archaeal community in the rhizosphere were less complex than they were in the bulk soil, and an unclassified archaeal group (Unclassified_k_norank) was identified as the keystone species in all archaeal networks.
\end{abstract}

Conclusions: Overall, the structure, assembly and co-occurrence patterns of archaeal communities are significantly affected by the presence of roots of alpine trees living on the Qinghai-Tibetan Plateau. This study provides new insights into our understanding of archaeal communities in vulnerable ecosystems.

Keywords: Rhizosphere, Archaeal community, Assembly process, Co-occurrence, Qinghai-Tibetan plateau

\section{Background}

The rhizosphere is a narrow zone of soil that tightly surrounds growing plant roots, which secrete a variable but substantial amount of photosynthesis-derived organic carbon compounds that enable the growth and metabolic activities of soil microorganisms [1,2]. Therefore, the rhizosphere has been considered to be one of the most complex interfaces in nature [3], where a variety of microorganisms drive multiple biogeochemical

\footnotetext{
* Correspondence: yhuang@pku.edu.cn

State Key Joint Laboratory of Environmental Simulation and Pollution Control, College of Environmental Science and Engineering, Peking University, No.5 Yiheyuan Road Haidian District, Beijing, P.R. China 10087
}

transformations including soil formation, carbon and nitrogen cycling $[4,5]$. In addition, rhizosphere microbial communities also have important effects on plant growth, health, and abiotic stress tolerance [6-8]. A growing number of studies have investigated the structure and assembly process of rhizosphere microbial communities, as well as their response to the selective effects of various biotic and abiotic factors [9-11]. However, these studies largely focused on bacteria and fungi, and little is known about the structural characteristics and driving factors of archaeal communities in the rhizosphere. 
In fact, archaea have been considered a substantial component of complex microbiomes [12], and have profound interactions with bacteria, fungi and viruses in a wide range of Earth's ecosystems [13, 14]. Compared to soil bacteria and fungi, archaeal communities are usually of low abundance and have less diversity [15], and they used to be thought to occur only in extreme environments [16]. Due to the rapid development of high-throughput sequencing technology, recent studies have expanded our knowledge of the biology of the archaea and have discovered their fundamental and even crucial ecological functions including methanogenesis [17], ammonia oxidation [18], hydrocarbon degradation [19], sulfate reduction [20], etc. Thus, a better knowledge of the structure, assembly and interaction of archaeal components in soil is of great importance [12].

Several studies have investigated the diversity and composition of archaeal communities in the rhizosphere. These studies are mainly limited to rice and a few wetland plants [21, 22], as well as only focusing on a minority of archaea taxa such as ammonia-oxidizing archaea (AOA) and methanogenic archaea [23-25]. Thus, it is not very clear what the diversity and composition of the archaeal community as a whole is in the rhizosphere, especially under unique environmental stress. In addition, the rhizosphere community structure is affected by the combination of environmental variables and interactions among microbial species [26, 27]. However, given the unique cellular structure and specific metabolic pathways of archaea that enable them to survive and even thrive under various adverse environments $[12,28]$, several key questions about the archaeal community also need to be answered. The first question is about the assembly process of archaea in rhizosphere: is it governed by a deterministic process or stochastic process? The second question is how archaeal species interact with one another. Co-occurrence network provides new perspectives for the analysis of microbiota beyond those of simple diversity and composition [29], and can well answer the second question.

The Qinghai-Tibetan Plateau (QTP), known as the "roof of the world" and "the third pole", is an important component of the terrestrial ecosystem, which provides many vital ecological services [30]. As one of the world's most vulnerable habitat, the QTP region has harsh environmental conditions and is highly sensitive to environmental disturbance, which could greatly impact the distribution of organisms, especially soil microorganisms [31-33]. The knowledge about their underground microorganisms have largely been studied [34-36], but the characteristics of rhizosphere microbiota, particularly archaeal communities are inadequate. In this study, high-throughput sequencing of $16 \mathrm{~S}$ rRNA gene amplicons was performed to exhaustively examine the archaeal communities derived from the rhizosphere of two native plants in the Qinghai-Tibetan Plateau. We aimed to investigate the effects of the rhizosphere of trees on the structure, assembly, and co-occurrence of archaeal communities in this ecologically vulnerable region. We tested the following hypotheses: 1) the archaeal community structure of tree rhizospheres are different from that of bulk soil in the QTP region; 2) the assembly of rhizosphere archaeal community are governed by deterministic processes in the QTP region; 3) the cooccurrence patterns of rhizosphere archaeal community are more complex than that of bulk soil in the QTP region.

\section{Results}

\section{Soil physicochemical properties}

The soil physicochemical properties significantly differed between the rhizospheres of two plant species and the bulk soil (Table 1; Table S1). The $\mathrm{pH}$ varied from 7.84 to 7.91, and the lowest $\mathrm{pH}$ was in the bulk soil. The moisture of the two plant rhizospheres were similar and were lower than that of the bulk soil. The highest content of soil organic matter (SOM) was observed in the bulk soil, and a significant difference was detected only in the rhizosphere of $P$. crassifolia compared to the bulk soil $(P<0.05)$. In addition, there were no significant differences in the content of total nitrogen (TN), alkalihydrolysable nitrogen (AN) and total phosphorus (TP) among the two plant rhizosphere and the bulk soil, but the content of ammonium nitrogen $\left(\mathrm{NH}_{4}{ }^{+} \mathrm{N}\right)$ and available phosphorus (AP) in the two rhizosphere were significantly higher than they were in the bulk soil $(P<$ 0.05).

\section{Diversity and community composition of archaea}

A total of 474,190 high-quality sequences were obtained with a median read count per sample of 39,516 (range: 30,420-54,538). The high-quality reads were clustered using $>97 \%$ sequence identity into 207 archaeal OTUs.

Table 1 Differences of soil physicochemical properties between two rhizosphere and bulk soils

\begin{tabular}{llll}
\hline Index & Bulk soil & P. crassifolia & P. szechuanica \\
\hline $\mathrm{pH}$ & 7.84(0.12)a & $7.87(0.05) \mathbf{a}$ & $7.91(0.04) \mathbf{a}$ \\
Moisture (\%) & $18.53(3.34) \mathbf{a}$ & $15.70(3.15) \mathbf{a}$ & $15.91(2.57) \mathbf{a}$ \\
$\mathrm{SOM}(\mathrm{g} / \mathrm{kg})$ & $32.63(2.82) \mathbf{b}$ & $25.55(3.97) \mathbf{a}$ & $27.68(4.42) \mathbf{a b}$ \\
$\mathrm{TN}(\mathrm{g} / \mathrm{kg})$ & $2.20(0.55) \mathbf{a}$ & $1.91(0.66) \mathbf{a}$ & $1.92(0.54) \mathbf{a}$ \\
$\mathrm{NH}_{4}{ }^{+} \mathrm{-N}(\mathrm{mg} / \mathrm{kg})$ & $13.88(0.76) \mathbf{a}$ & $15.44(0.62) \mathbf{b}$ & $15.55(0.31) \mathbf{b}$ \\
$\mathrm{AN}(\mathrm{mg} / \mathrm{kg})$ & $255.75(67.09) \mathbf{a}$ & $236.50(75.24) \mathbf{a}$ & $261.25(47.25) \mathbf{a}$ \\
$\mathrm{TP}(\mathrm{g} / \mathrm{kg})$ & $0.34(0.03) \mathbf{a}$ & $0.36(0.03) \mathbf{a}$ & $0.35(0.03) \mathbf{a}$ \\
AP $(\mathrm{mg} / \mathrm{kg})$ & 5.50(0.27)a & $6.03(0.33) \mathbf{b}$ & $6.03(0.28) \mathbf{b}$ \\
\hline
\end{tabular}

Data are means \pm SD in parentheses, and different letters in the columns indicate significant differences (Dunnett test, $P<0.05$ ) 
The Good's coverage scores (in all cases above 99.9\%) and the rarefaction curves showed clear asymptotes (Fig. S1), which together indicated a near-complete sampling of the archaeal community in this study.

The diversity indices of archaeal communities varied among the rhizospheres of two plant species and the bulk soil (Table 2). The observed number of OTUs $(\mathrm{Ob})$ was highest in bulk soil, followed by the rhizosphere of $P$. szechuanica, whereas the rhizosphere of $P$. crassifolia had lower numbers. Conversely, the Shannon index in the two plant rhizospheres were higher than they were in the bulk soil, and significant difference was identified only in the rhizosphere of $P$. szechuanica compared to the bulk soil $(P<0.05)$. The phylogenetic diversity (MNTD) of the two plant rhizospheres were similar, and their values were higher than that of the bulk soil.

Principal coordinate analysis ( $\mathrm{PCoA})$ based on weighted UniFrac distances was performed to investigate the patterns of separation among archaeal microbiota. We clearly observed strong clustering of archaeal communities according to the different microhabitats (i.e., P. crassifolia, P. szechuanica rhizosphere and bulk soil). Moreover, the two plant rhizosphere samples were clearly distinguished from the bulk soil samples across the first principal coordinate, while the separation between the rhizosphere of $P$. crassifolia and $P$. szechuanica was seen along the second principal coordinate, indicating that the largest source of variation in the archaeal communities is proximity to the root, followed by plant variety (Fig. 1a). Interestingly, PCoA analysis of $\beta M N T D$ distances revealed that the largest source of variation is plant variety rather than proximity to the root (Fig. S2). Consistent with the result of PCoA analyses, ANOSIM analyses also revealed significant differences in the structure of archaeal communities among the rhizosphere of two plant species and the bulk soil (Table S2).

The relative abundance of archaeal OTUs at the phylum level was variable among the two plant rhizospheres and the bulk soil. The most dominant archaeal phyla across all samples were Thaumarchaeota, Unclassified_k_norank and Euryarchaeota, accounting for 92.46-98.01\%, 1.35$6.01 \%$ and $0.56-1.18 \%$ of the pyrosequencing reads, respectively (Fig. 1b). Analysis of variance (ANOVA) showed significant enrichment of Thaumarchaeota in the rhizosphere microbiota of two plant species compared to

Table 2 Differences in the a-diversity indices of archaeal communities between two rhizosphere and bulk soils

\begin{tabular}{|c|c|c|c|}
\hline & \multicolumn{2}{|c|}{ Taxonomic Diversity } & \multirow{2}{*}{$\begin{array}{l}\text { Phylogenetic Diversity } \\
\text { MNTD }\end{array}$} \\
\hline & $\mathrm{Ob}$ & Shannon & \\
\hline Bulk soil & 69(23)a & $2.06(0.15) \mathbf{a}$ & $0.36(0.11) \mathbf{a}$ \\
\hline P. crassifolia & $45(14) \mathbf{a}$ & 2.23(0.11)ab & $0.43(0.22) \mathbf{a}$ \\
\hline P. szechuanica & $63(14) \mathbf{a}$ & $2.34(0.03) \mathbf{b}$ & $0.44(0.06) \mathbf{a}$ \\
\hline
\end{tabular}

Data are means \pm SD in parentheses, and different letters in the columns indicate significant differences (Dunnett test, $P<0.05$ ) that of the bulk soil (Dunnett test, $P<0.05$ ). Conversely, the relative abundance of Unclassified_k_norank and Euryarchaeota in the rhizosphere microbiota of two plant species decreased but did not show significant differences compared with the abundance in the bulk soil (Table S3). Moreover, LEfSe analysis was also performed to determine the taxa that most likely explains the variations among different samples. In the bulk soil, four groups of archaea were significantly enriched, namely, Thermoplasmata (the class, orders of Thermoplasmatales, and its family marine_Group_II to genus), unclassified_k_norank (from phylum to genus), norank_c_Soil_Crenarchaeotic_Group_ SCG (from order to genus), group_C3 (from family to genus). In the $P$. crassifolia rhizosphere, a group of archaea was significantly enriched, namely, Thaumarchaeota (the phylum and its class soil_Crenarchaeotic). In the $P$. szechuanica rhizosphere, two groups of archaea were significantly enriched, namely, unclassified_c_Soil_Crenarchaeotic_Group_SCG (from order to genus), unknown Order_c_Soil_Crenarchaeotic_Group_SCG (from order to genus) (Fig. 2).

\section{Correlation between soil properties and archaeal communities} Distance-based redundancy analysis (dbRDA) indicated the strong correlation between soil physicochemical characteristics and the structure of archaeal communities. The first two axes of CAP could explain 27.12 and $13.43 \%$ of the total variation in archaea communities, respectively (Fig. 3). In line with the PCoA (weighted UniFrac) analysis, the first axis (CAP1) could separate the rhizosphere samples from the bulk soil, and the second axis (CAP1) mainly distinguished the $P$. crassifolia rhizosphere from the P. szechuanica rhizosphere samples. The results of PERMANOVA analysis revealed that soil ammonium nitrogen $\left(\mathrm{NH}_{4}{ }^{+}-\mathrm{N}\right)$, soil organic matter (SOM) accounted for 35.1 and $28.5 \%$ of archaeal community differences, respectively, and niches (rhizosphere vs bulk soil) contributed $45.4 \%$ of the interpretation (Table S4). In addition, soil ammonium nitrogen $\left(\mathrm{NH}_{4}{ }^{+} \mathrm{N}\right)$, available phosphorus (AP) and $\mathrm{pH}$ value were important environmental attributes significantly affecting the archaea community structure (Mantel test; $r=0.392$, $P=0.026 ; r=0.362, P=0.030 ; r=0.400, P=0.028$ ).

Further analyses revealed that soil properties had significant effects on the relative abundance of the archaea taxa at the class level. Soil pH value was positively correlated with the relative abundance of Unclassified_k_norank, Norank_p_Bathyarchaeota, and it was negatively correlated with Soil_Crenarchaeotic_Group_ SCG, Methanobacteria. Ammonium-nitrogen $\left(\mathrm{NH}_{4}{ }^{+}-\mathrm{N}\right)$ was positively correlated with the relative abundance of Thermoplasmata. Soil total phosphorus (TP) was positively correlated with the relative abundance of Methanobacteria. Available phosphorus (AP) was negatively correlated with Unclassified_k_norank (Fig. 4). 

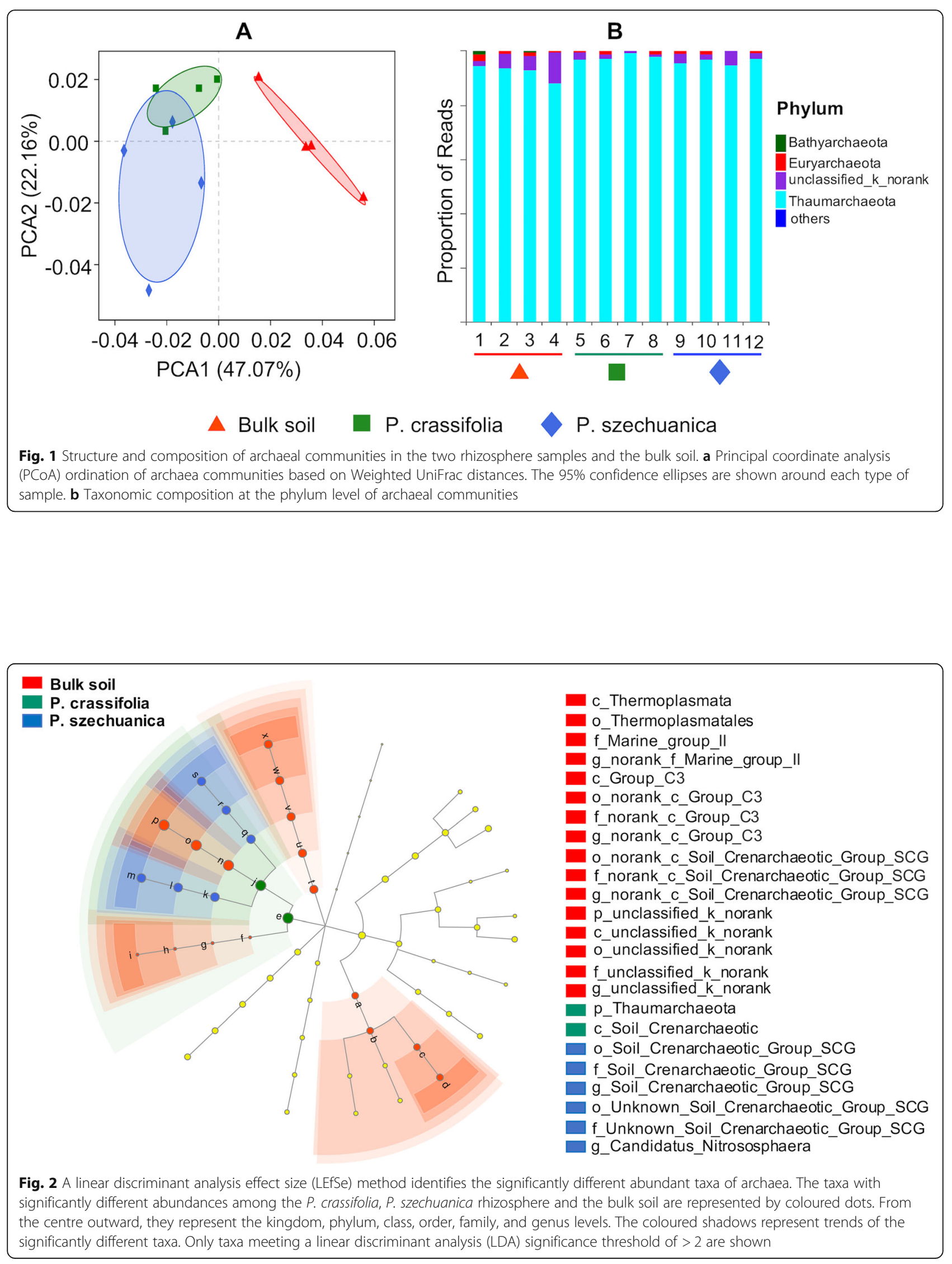


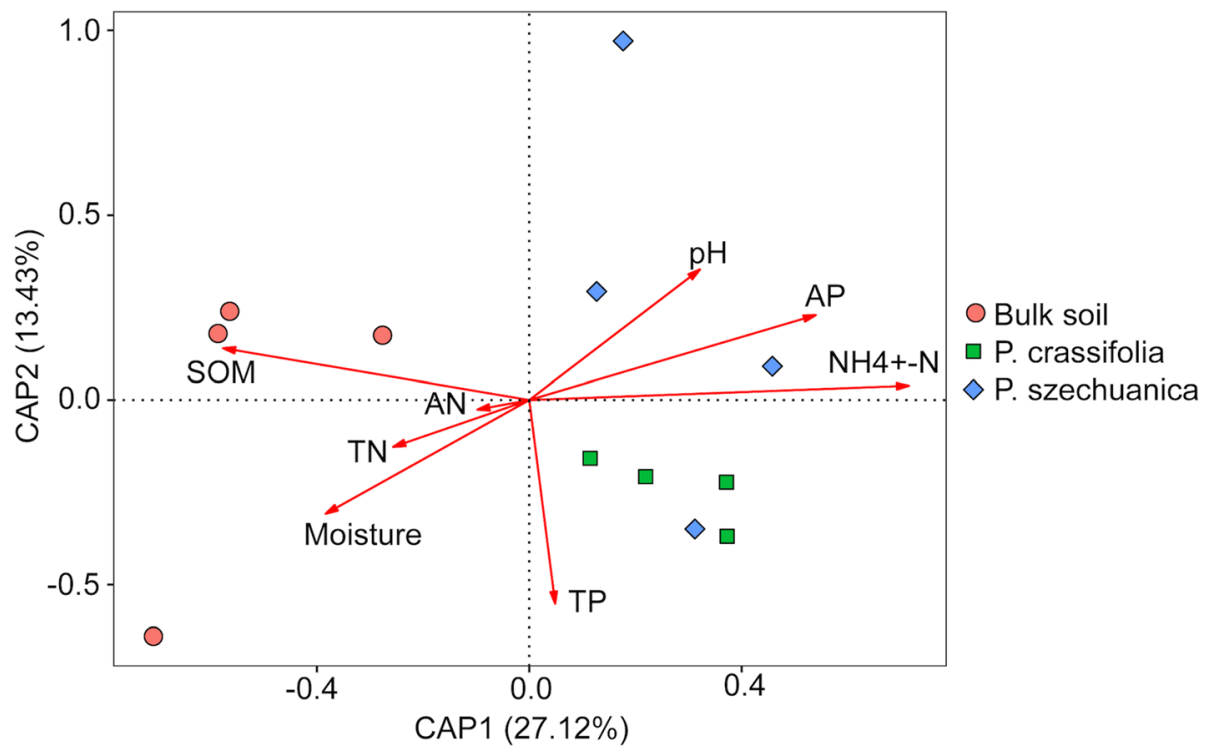

Fig. 3 Ordination plots of the results from distance-based redundancy analysis (dbRDA) to explore the correlation between soil properties and archaeal community structure

\section{Assembly processes of archaeal microbiota in rhizosphere and bulk soil}

The phylogenetic tree of archaea recovered from all samples were relatively well classified according to the major lineages, and the local support values on the branches were relatively high (Fig. S3), suggesting the archaeal phylogenetic tree was reliable. Additionally, the phylogenetic signal showed that there was a significant relationship between ecological similarity and phylogenetic relatedness across short phylogenetic distances (Fig. S4). Thus, we calculated NTI and $\beta$ NTI because both of these metrics emphasize phylogenetic relationships across short phylogenetic distances [37].

We clearly observed that the NTI values of archaea microbiota from all samples were less than -2 , in which the lowest mean NTI value was detected in the bulk soil (Fig. 5a), suggesting that archaeal communities were phylogenetically over-dispersed, especially in the bulk soil, and it also suggested that deterministic processes mainly regulate the

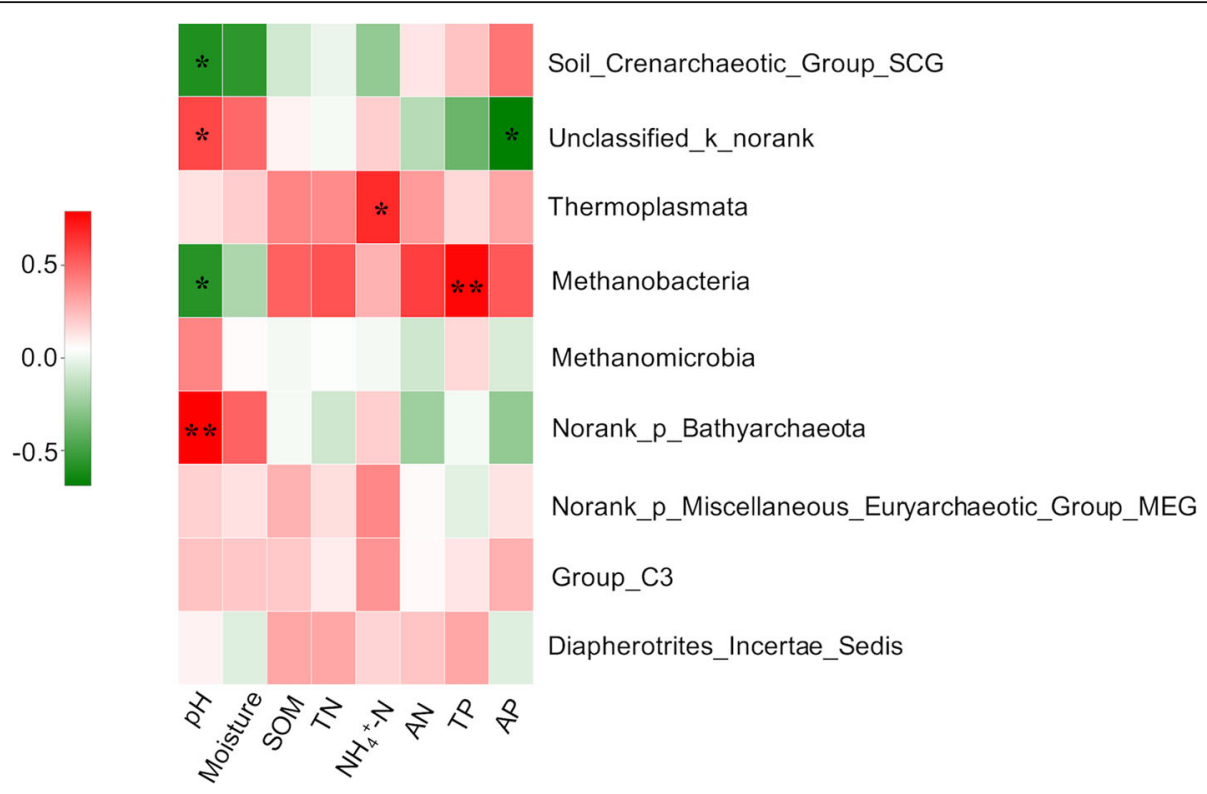

Fig. 4 Heat map showing the Pearson correlation between soil properties and the relative abundance of the archaea taxa at the class level. The left side of the legend is the colour range of different R-values. The value of $P<0.05$ and $P<0.01$ is marked with "** and "**", respectively 

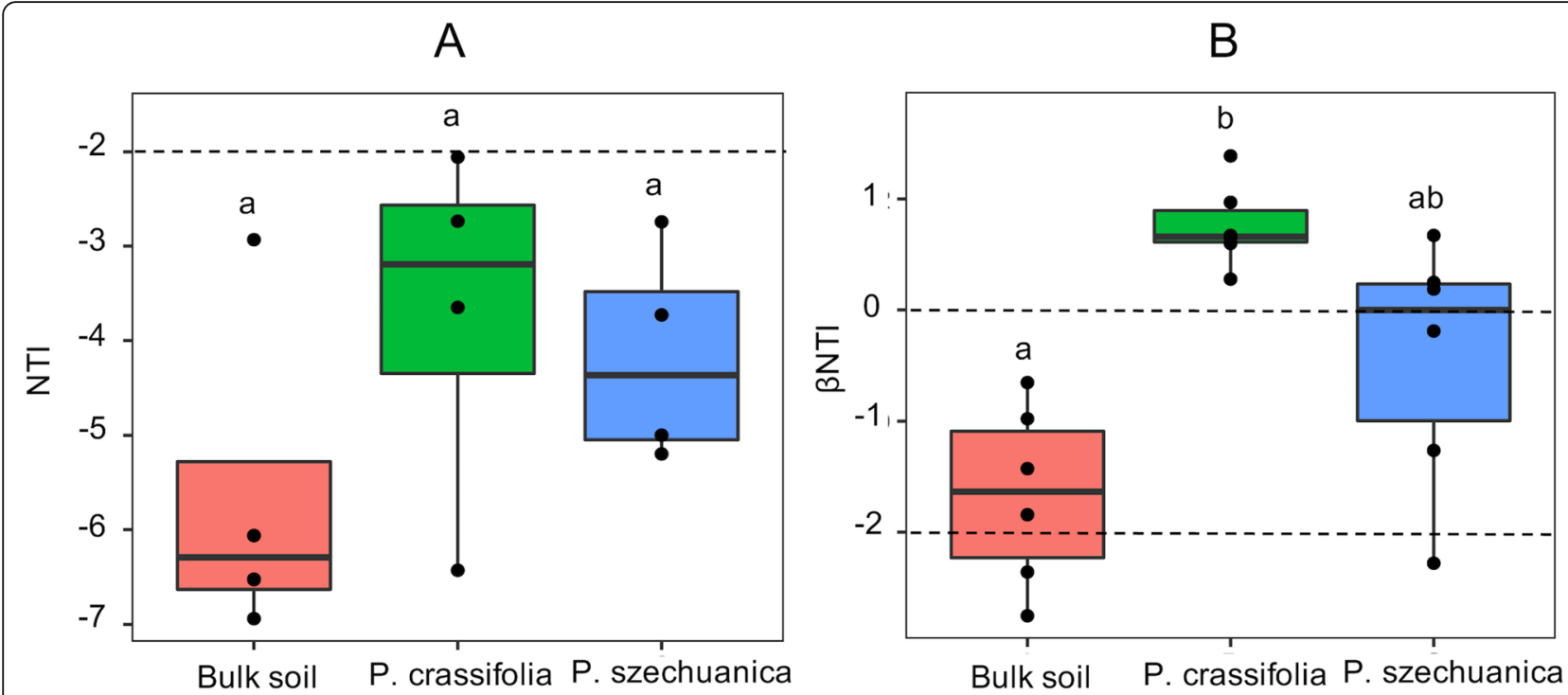

Fig. 5 Box plot of NTI metric (a) and BNTI metric (b) of archaeal communities in all samples. Letters above the boxes indicate significant differences (Dunnett test, $P<0.05$ )

assembly of archaeal communities. In addition, the lowest mean $\beta$ NTI value for archaea was found in the bulk soil, and it was significantly lower than zero (66.67\% of $\beta$ NTI values ranging between 0 and $-2,33.33 \%$ of $\beta$ NTI values less than -2 ), suggesting the phylogenetic turnover was less than what would be expected by chance. The mean $\beta$ NTI value in the $P$. crassifolia rhizosphere was significantly higher than zero, suggesting the phylogenetic turnover was higher than what would be expected by chance. However, the mean $\beta$ NTI value in the $P$. szechuanica rhizosphere was not significantly different from zero (Fig. 5b). These results further indicated that deterministic processes play a stronger role in the phylogenetic turnover than stochastic processes. Additionally, the species rank abundance distribution models also revealed that archaea communities in all samples were followed 'niche theory' models (Table 3).

\section{Co-occurrence network structure of archaea microbiota}

Three co-occurrence networks were constructed for all sample types (Bulk soil, P. crassifolia and P. szechuanica

Table 3 The proportion of the lowest AIC values for six species rank abundance distribution models of archaea communities in all samples

\begin{tabular}{lccc}
\hline & Bulk soil & P. crassifolia & P. szechuanica \\
\hline Break Stick & & & \\
Pre-emption & $75 \%$ & $100 \%$ & $75 \%$ \\
Lognormal & & & \\
Zipf & & \\
$\quad$ Zipf-Mandelbrot & $25 \%$ & & \\
ZSM & & & \\
\hline
\end{tabular}

The blank cells indicate ' $0 \%$ ' rhizosphere) to illustrate potential biotic interactions among archaea taxa (Fig. 6). All the networks were significantly different from the random networks with the identical numbers of nodes and edges (Table S5), suggesting that the network structures were nonrandom and reliable.

We found that the networks in the rhizosphere of two tree species were obviously different from that in the bulk soil (Fig. 6; Table S5). The number of edges, average degree and average clustering coefficient of the networks in two rhizosphere were lower than they were in bulk soil, indicating that rhizosphere assemblages of two plant species formed lower complex archaea networks compared with that of the bulk soil. The ratio of negatively correlated edges between OTUs in the $P$. crassifolia rhizosphere $(30.8 \%)$ and the $P$. szechuanica rhizosphere (27.6\%) were profoundly higher than that of in bulk soil (20.1\%), which could be interpreted as increased competitions among archaea taxa in the rhizosphere environment. We also observed a high proportion of unclassified_k_norank in all networks (Bulk soil, $P$. crassifolia and $P$. szechuanica rhizosphere), accounting for 52.0, 52.0 and $62.0 \%$, respectively. In addition, the majority of unclassified_k_norank were highly connected in the networks. Thus, it could be inferred that unclassified_k_norank is very crucial for the stability of archaea network structures in all samples.

\section{Discussion}

\section{Variation of archaea community structures between} rhizosphere and bulk soil

In this study, the structure of archaeal communities in the rhizosphere of two tree species were significantly 


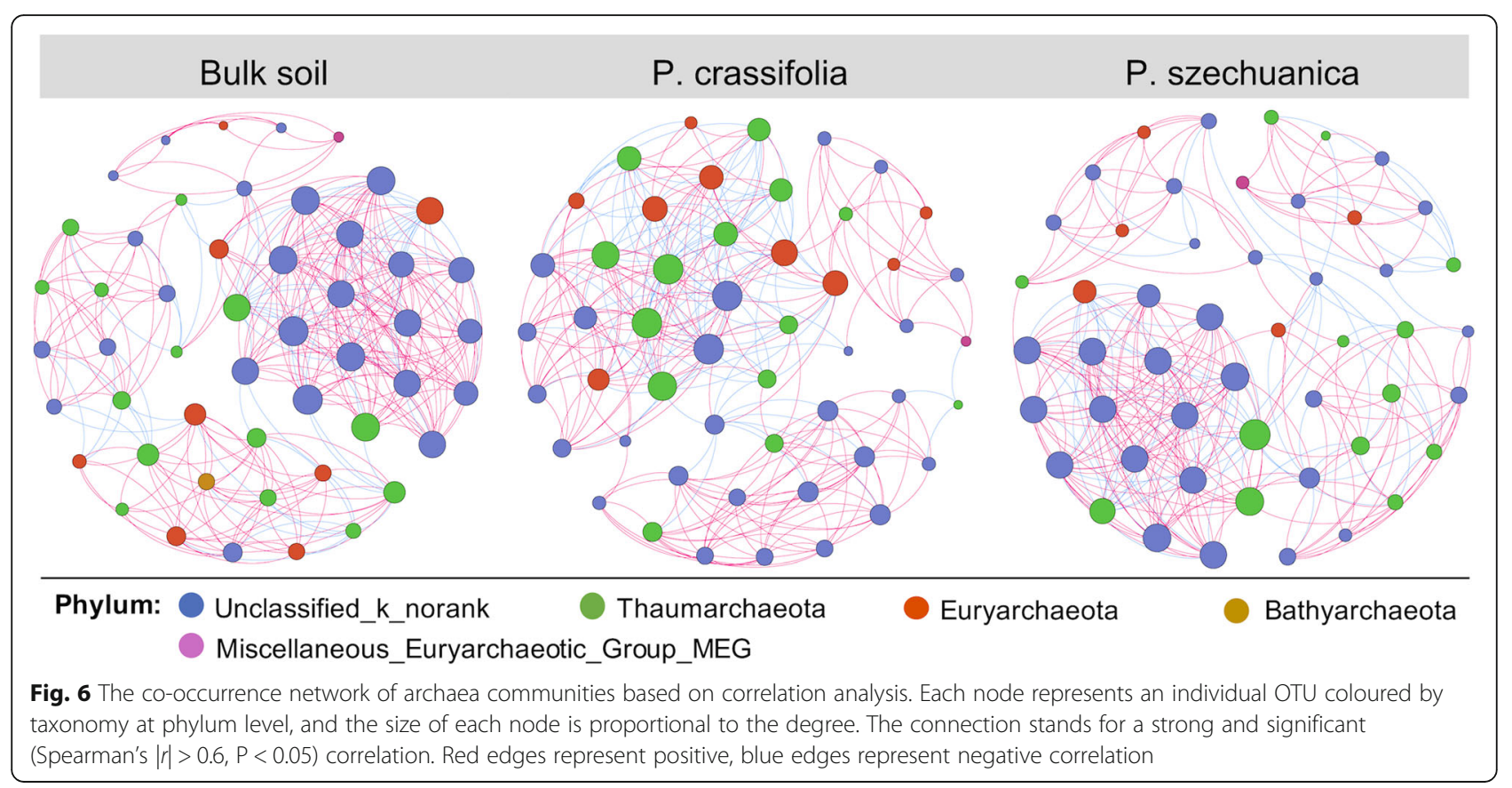

different from that in the bulk soil in the QTP region (Fig. 1; Fig. S1; Table 2; Table S2), which supported our first hypothesis. Moreover, we found that the largest source of variation in archaeal communities is the presence of plant roots, followed by plant species, which agreed with previous studies of rhizosphere bacterial and fungal communities [38-41]. Plant roots could release a variety of carbon exudates including sugars, amino acids, organic acids, mucilage and root border cells [42, 43]. These exudates are available nutrients and energy for microbial activities [44], making the microbial community structures in the rhizosphere differed from what is found in the bulk soil [5]. The variation of archaeal communities observed between the rhizosphere $P$. crassifolia and $P$. szechuanic may be due to the difference in the composition of carbon exudates released by the roots of the two tree species [45]. In addition, the plant rhizospheres could form oxygen-depleted micro-niches for soil microorganisms due to the respiration of the roots [46]. Most of Archaea have also been identified as strictly anaerobic or facultative anaerobes [12], which are likely to be affected by the changes of redox potential in the rhizosphere. Consistent with this inference, important groups of Archaea, such as ammoniumoxidizing archaea and methanogens, have been proved to have unique distribution in the rhizosphere of Phragmites australis and Halocnemum strobilaceum [25, 47]. Therefore, this may also explain the difference of archaeal communities between the rhizosphere and bulk soil in the QTP region.

Analysis of archaeal community composition revealed that the archaeal communities were dominated by
Thaumarchaeota phylum, accounting for 92.46-98.01\% of sequences in this study (Fig. 1b; Table S3). This finding was in agreement with previous findings from the research also conducted in the Qinghai-Tibetan Plateau, which showed that the dominant archaeal phylum was Thaumarchaeota, accounting for $79.27 \%$ of sequences [32]. Thaumarchaeota have been detected in a variety of habitats [48-50], and identified as a novel archaeal phylum in 2008 [48]. Many studies have suggested that Thaumarchaeota species possess ammonia oxidizing abilities and are considered to play an important role in nitrogen cycling [51, 52]. In our study, the relative abundance of Thaumarchaeota in the two different plant rhizosphere were significantly higher than they were in the bulk soil (Fig. 1b; Table S3), and all the eight biomarkers in rhizosphere by the LEfSe analysis also belong to the phylum Thaumarchaeota (Fig. 2). These findings collectively indicated that the nitrogen metabolism activities occurred in the rhizosphere might be higher than that in that in the bulk soil.

\section{Important drivers of archaea communities}

Combined with the analysis of dbRDA and Mantel test showed that soil ammonium nitrogen $\left(\mathrm{NH}_{4}{ }^{+}-\mathrm{N}\right)$, available phosphorus (AP) and $\mathrm{pH}$ were significantly correlated with the archaeal community structures (Fig. 3). This observation in $\mathrm{NH}_{4}{ }^{+}-\mathrm{N}$ agreed with previously reported results in the study by Norman and Barrett [53], which documented $\mathrm{NH}_{4}{ }^{+}-\mathrm{N}$ as a metabolic substrate that drives the distribution patterns in richness of ammonia-oxidizing archaea (AOA). Moreover, our results revealed that the content of $\mathrm{NH}_{4}{ }^{+}-\mathrm{N}$ in the 
rhizosphere was significantly higher than it was in the bulk soil (Table 1), and the increase of $\mathrm{NH}_{4}{ }^{+}-\mathrm{N}$ concentration in the rhizosphere may be due to the enrichment of diazotrophic bacteria in the rhizosphere, which can convert atmospheric $\mathrm{N}_{2}$ into ammonium via biological nitrogen fixation $[54,55]$, although the results of this study cannot be directly confirmed. The relative abundance of the class Thermoplasmata was positively correlated with soil $\mathrm{NH}_{4}{ }^{+}-\mathrm{N}$ (Fig. 4). These results corroborate the opinion that $\mathrm{NH}_{4}{ }^{+}-\mathrm{N}$ may play a significant role in shaping the archaeal community structures. $\mathrm{Nu}$ merous studies have suggested that soil $\mathrm{pH}$ is a major driver of the community structure of bacteria, fungi or diazotrophs [56-58], but there seems to be no consensus on archaea [53,58]. This contradiction could be explained by a plausible interpretation: soil $\mathrm{pH}$ indirectly affects the abundance of major archaeal taxa mainly by regulating the availability of substrates such as $\mathrm{NH}_{4}{ }^{+}$, $\mathrm{CO}_{2}$, and $\mathrm{CH}_{3} \mathrm{COOH}[53,59,60]$, so the correlation may vary with samples or by region. The significant correlation in our study could be attributed to more available $\mathrm{NH}_{4}{ }^{+}-\mathrm{N}$ regulated by $\mathrm{pH}$ to the phylum Thermoplasmata [61]. Soil available phosphorus (AP) has been reported to be a limiting factor for the growth of plants or microorganisms [7, 62]; thus, it may directly or indirectly affect archaeal communities.

\section{Deterministic processes govern the assembly of archaeal communities}

Our results indicate that the assembly of archaeal communities were governed by the deterministic processes across all soils in the QTP region (Fig. 5; Table 3), consistent with our second hypothesis. The mean NTI values were significantly lower than zero in all samples, which provided concrete evidence that the archaeal communities were more phylogenetically over-dispersed than expected as a result of chance [63, 64]. Previous studies have shown that the competition among species would become more frequent where there was greater niche similarity and would subsequently lead to the coexistence of distantly phylogenetically related species $[65,66]$. In the present study, most of the soil variables (except for $\mathrm{NH}_{4}{ }^{+}-\mathrm{N}$ and $\mathrm{AP}$ ) were similar (Table 1), and microorganisms competing strongly for nutrients or water in the QTP region suffered from its low temperature and strong ultraviolet radiation [67, 68], which was supported by the high proportion of negative interactions in archaeal networks (Fig. 6; Table S5). These factors may explain why the archaeal communities were phylogenetically over-dispersed. Furthermore, the NTI values in the rhizosphere were greater than that in the bulk soil but not significant, which might indicate that stochastic processes may still play a minor role [69]. In fact, former researches have already proved that the assembly of ecological communities are regulated concurrently by both stochastic and deterministic processes $[37,70,71]$. In addition, We also found that the rhizosphere $\beta$ NTI values were significantly greater than those measured in the bulk soil, suggesting that the phylogenetic turnover of archaea in the rhizosphere were higher than what was in the bulk soil $[55,71]$. This could be attributed to dynamic rhizosphere microhabitats potentially stimulating the activities and evolutions of archaeal species [3].

\section{Distinct archaeal networks in rhizospheres and bulk soil}

Previous studies have found that bacterial or fungal networks in the rhizospheres were more $[40,72]$ or less $[27,73]$ complex than what were found in the bulk soil. In the present study, we found that the archaeal cooccurrence networks of in the rhizosphere of two tree species were less complex relative to that of the bulk soil in the QTP region (Fig. 6; Table S5), which not supported our third hypothesis. Considering the complexity of microbial networks may represent ecological interactions or niche sharing among microorganisms [74], the rhizosphere of two tree species in QTP region likely fosters fewer archaeal interactions or develops less shared niches than the bulk soil, which could be explained through two plausible interpretations. On the one hand, the archaea possess distinctive metabolic pathways and enzymes that enable them to survive and thrive under extreme or nutrient-poor environments $[12,13]$, which may lead to their lower nutrient dependence on root exudates than bacteria or fungi. On the other hand, the rhizosphere bacterial and fungal species are likely to accelerate the consumption of substrates required by archaea, and even the plants themselves may be competitors for microorganisms under severe environmental stress in the QTP region [75, 76], thus reducing the interactions or niche sharing among archaea. This interpretation was also supported by the finding that higher numbers of negative links occurred in the rhizosphere networks. Moreover, average path length of archaeal network was smaller in the rhizospheres than in the bulk soil. Networks with small path length are considered to be small-world networks [77], which are related to the rapid responses of ecosystems to perturbations [78]. Therefore, archaeal community in the tree rhizospheres may be more sensitive to climate change compared with in the bulk soil in ecologically vulnerable region. We also found that an unclassified archaeal group, unclassified_k_norank occupies a high proportion in all networks despite its low abundance in the community composition (Fig. 1b; Table S3). Even though we do not yet know the specific ecological functions of this unclassified archaeal group, it is clear that it may play an 
important role in maintaining the stability of community structure and function [79].

\section{Conclusions}

In summary, our study provides insight into the structure, assembly and co-occurrence patterns of the rhizosphere archaeal communities in the QTP region. The results showed that archaeal community structures in the rhizosphere of two plant species significantly differed from that in the bulk soil. Soil ammonium-nitrogen $\left(\mathrm{NH}_{4}{ }^{+}-\mathrm{N}\right)$, soil organic matter $(\mathrm{SOM})$, available phosphorus (AP) and $\mathrm{pH}$ were important drivers of the archaeal communities. Deterministic processes dominated the assembly of archaeal communities across all samples. The network structures of the archaeal community in the rhizosphere were less complex than they were in the bulk soil. We also identified an unclassified archaeal group (unclassified_k_norank) that may be crucial for the interrelationships among archaeal species. Future research should further investigate the interaction between archaea and other microorganisms such as bacteria, fungi and protists in the rhizosphere, and work to understand the role of archaea in plant survival and growth under low-temperature stress.

\section{Methods}

\section{Site and sampling}

A trees field trial located in the northeast portion of the Qinghai-Tibetan Plateau $\left(31^{\circ} 32^{\prime} \mathrm{N}, 92^{\circ} 00^{\prime} \mathrm{E}, 4531 \mathrm{~m}\right.$ a. s. l), which has a plateau sub-frigid monsoon semi-arid climate with an average annual temperature of $-2.2^{\circ} \mathrm{C}$ and a mean annual precipitation of $458 \mathrm{~mm}$. This field trial was established in April 2010 and contains two native alpine tree species (Picea crassifolia and Populus szechuanica var. tibetica). These two woody plants represent the typical coniferous and broad-leaved plants living in the QTP area, respectively.

In order to ensure the representativeness of the samples, the surviving and well-growing trees $(P$. crassifolia about $2.5 \mathrm{~m}$, P. szechuanica about $4.5 \mathrm{~m}$ ) were selected for sample collection in July 2017. Three subsamples of fine roots $(<2 \mathrm{~mm})$ were carefully collected from different positions in the rhizosphere of each selected tree at the depth of $5-15 \mathrm{~cm}$ below ground level. The homogeneous rhizosphere soil was obtained from the combined fine root samples of each tree according to the procedure described in a previous study [38]. The bulk soil was collected from four treeless quadrats $(3 \mathrm{~m} \times 3 \mathrm{~m})$ at the depth of $5-15 \mathrm{~cm}$ below ground level. Each quadrat is about $10 \mathrm{~m}$ away from the sampled trees, in which five soil subsamples were obtained and combined into a representative bulk soil sample. All soil samples were handpicked to remove roots and impurities, and then divided into two subsamples. One portion was air dried and sieved through $2 \mathrm{~mm}$ meshes for soil property analyses, and the other portion was stored at $-80^{\circ} \mathrm{C}$ for DNA extraction.

\section{Soil physicochemical properties analysis}

Soil physicochemical properties in both rhizosphere and bulk soils were analysed according to Zhou et al. [80]. Briefly, soil moisture was quantified gravimetrically by drying fresh soils in $105^{\circ} \mathrm{C}$ for $48 \mathrm{~h}$. Soil pH was measured by a $\mathrm{pH}$ meter in a soil suspension with an air-dried soil to water radio of 1: 2.5 mass/volume. Soil organic matter (SOM) was determined by the potassium dichromate oxidation titration method. Total nitrogen (TN) was measured by the Kjeldahl digestion method. Total phosphorus (TP) was determined by the Mo-Sb anti-spectrophotometric method. Alkali-hydrolysable nitrogen (AN) was measured by the alkali-hydrolysed diffusing method. Ammonium nitrogen $\left(\mathrm{NH}_{4}{ }^{+}-\mathrm{N}\right)$ was measured using indophenol blue spectrophotometry. Available phosphorus (AP) was extracted with a $\mathrm{NH}_{4} \mathrm{~F} /$ $\mathrm{HCl}$ solution, which was then determined using a UVvisible spectrophotometer.

\section{DNA extraction, PCR amplification, and sequencing}

Total genomic DNA was extracted using the DNeasy PowerSoil Kit (Qiagen, Hilden, Germany) according to the manufacturer's instructions. The quantity and quality of DNA was evaluated with a spectrophotometer (NanoDrop, ND2000, Thermo Scientific, Wilmington, DE, USA). The primers 524F10extF ( $5^{\prime}$-TGYCAGCCGC CGCGGTAA-3') and Arch958RmodR (5'-YCCGGCGT TGAVTCCAATT-3') were chosen for the amplification and subsequent high-throughput sequencing of the archaea [81]. Each sample was amplified in triplicate in a $20 \mu \mathrm{L}$ mixture containing $4 \mu \mathrm{L}$ of $5 \times$ FastPfu Buffer, $2 \mu \mathrm{L}$ of $2.5 \mathrm{mM}$ dNTPs, $0.8 \mu \mathrm{L}$ of each primer $(5 \mu \mathrm{M}), 0.4 \mu \mathrm{L}$ of FastPfu Polymerase and 10 ng of template DNA. The PCR reaction were carried out using the following protocol: 3 min of denaturation at $95^{\circ} \mathrm{C}, 27$ cycles of $30 \mathrm{~s}$ at $95^{\circ} \mathrm{C}, 30 \mathrm{~s}$ of annealing at $55^{\circ} \mathrm{C}, 45 \mathrm{~s}$ of elongation at $72^{\circ} \mathrm{C}$, and a final extension at $72{ }^{\circ} \mathrm{C}$ for $10 \mathrm{~min}$. The PCR products were extracted and purified by agarose gel electrophoresis, and further quantified using QuantiFluor $^{\text {rw }}$-ST (Promega, USA) according to the manufacturer's protocol. Purified amplicons were pooled in equimolar amounts and paired-end sequencing was performed on an Illumina MiSeq platform (Illumina, San Diego, USA) according to the standard protocols described by Majorbio Bio-Pharm Technology Co. Ltd.

\section{Sequence processing}

Raw sequences yielded from Illumina sequencing were processed using QIIME 1.9.1 [82]. Paired-end reads were joined with fastq-join, demultiplexed and quality filtered 
with default parameters [83]. Briefly, sequences with a quality score $<20$ or with any truncated reads shorter than $50 \mathrm{bp}$ were removed. Operational taxonomic units (OTUs) were clustered with $97 \%$ similarity cutoff using UPARSE 7.1 and chimeric sequences were identified and removed using UCHIME. The taxonomy of each $16 \mathrm{~S}$ rRNA gene sequence was analysed by an RDP Classifier algorithm (http://rdp.cme.msu.edu/) against the Silva (SSU123) 16S rRNA database using a confidence threshold of $80 \%$.

\section{Data analysis}

All statistical analyses were carried out using R (v 3.5.1, The $\mathrm{R}$ Core Team, 2018) unless stated otherwise. $\alpha$ diversity in each sample was calculated as the observed number of OTUs $(\mathrm{Ob})$, the Shannon diversity and the phylogenetic diversity (MNTD) indices. Significant differences in the variance of $\alpha$-diversity and microbial abundance data were examined using one-way analysis of variance, and post hoc comparisons were conducted by the Dunnett test at the $5 \%$ level. The differences in archaeal community composition based on Weighted UniFrac and $\beta M N T D$ distances were illustrated with PCoA ordination plots using the 'cmdscale' function from the vegan package. To statistically support the archaeal clustering patterns resulted from PCoA analysis, different samples were compared by ANOSIM analysis using the vegan package. Additionally, we performed linear discriminant analysis (LDA) coupled with effect size measurements (LEfSe) analysis to investigate statistically representative biomarkers between different samples.

Distance-based redundancy analysis (dbRDA) was carried out using the 'rda' function from the vegan package to explore the relationships between soil physicochemical properties and archaeal community composition. Furthermore, associations between soil properties and nine archaeal classes were evaluated by Pearson correlation analysis at the $5 \%$ level.

To evaluate the assembly processes of the archaeal community, the phylogenetic signal of each sample was first tested by following the procedure described by a previous study [69]. Briefly, environmental optima for each OTU with respect to all physicochemical variables were calculated. The correlation coefficients between phylogenetic distances and differences in environmental optima were calculated by phylogenetic Mantel correlograms [55], and the significance of these correlations were examined with 999 randomizations using the 'mantel.correlog' function from the vegan package. The phylogenetic diversity within each sample was calculated as the mean nearest taxon distance (MNTD) and nearest taxon index (NTI) using the 'mntd' and 'ses.mntd' functions from the picante package [84] Note that MNTD refers to the phylogenetic distance between each OTU and its closest relative also found per sample, and NTI measures the deviation of observed MNTD from MNTD in a null model with 999 randomizations. For NTI $>+2$ $(\mathrm{NTI}<-2)$ in a single community or a mean NTI $>0$ $(\mathrm{NTI}<0)$ significantly across all communities indicates coexisting taxa are more closely related (phylogenetic clustering) or more distantly related (phylogenetic overdispersion) than can be expected by chance [37]. The pairwise phylogenetic turnover between communities was calculated as $\beta$ MNTD and $\beta$ NTI using the 'comdistnt' function from the vegan package [84]. $\beta$ NTI $>+2$ $(\beta \mathrm{NTI}<-2)$ between one pair of communities or mean $\beta$ NTI $>0(\beta$ NTI $<0)$ significantly in all pairs of communities indicates greater (or less) than expected phylogenetic turnover, respectively [69]. If the observed BMNTD values does not significantly deviate from the null $\beta M N T D$ distribution [85], it suggests that stochastic processes predominate phylogenetic community composition. In addition, to verify the results from phylogenetic analyses, five models representing niche theory (Break Stick, Pre-emption, Lognormal, Zipf, ZipfMandelbrot) and ZSM representing neutral theory were performed using the function 'radfit' from the $\mathrm{R}$ package 'vegan' or TeTame [64]. Akaike Information Criterion (AIC) was used to evaluate the fitting quality of each statistical model, where the lower AIC value indicated a better fit for the model [86].

Network analyses based on Spearman's rank analysis were carried out with the 'WGCNA' package [30, 87], and structural attributes of the overall networks including average degree, clustering coefficient and average path distance were calculated in the 'igraph' package. The 50 most abundant OTUs of the archaea community in each sample were selected, and the co-occurrence patterns of archaea communities were explored based on strong and significant correlation (Spearman's $|r|>$ $0.6, P<0.05)$. Finally, the constructed networks were visualized using Gephi 0.9.2 [88].

\section{Supplementary information}

Supplementary information accompanies this paper at https://doi.org/10. 1186/s12866-020-01913-5.

Additional file $\mathbf{1}$ Table S1. Soil physicochemical properties of the rhizosphere and bulk soils. Data are means \pm SD in parentheses, and different letters in the columns indicate significant differences $(P<0.05)$. Table S2. ANOSIM analyses of separable compartments on archaeal community beta diversity distance matrix. P. crassifoli and P. szechuanica means the rhizosphere soil of Picea crassifolia and Populus szechuanica. Table S3. Comparison of sample differences in abundance of phyla. Data are means \pm SD in parentheses. Different letters indicate significant levels (Dunnett test, $P<0.05$ ). For abbreviations, see Table S1. Table S4 Factors affecting the structure of archaeal communities in the rhizosphere and the bulk soil revealed by PERMANOVA. NS means not significant. Table S5. Co-occurrence network topological features statistics in three compartments. For abbreviations, see Table S1. Figure S1. Rarefaction curves comparing the number of sequences with the number 
of observed OTUs for archaeal communities in each sample. For abbreviations, see Table 1. Figure S2. Principal coordinate analysis (PCOA) ordination of archaeal communities based on MNTD index. For abbreviations, see Table 1. Figure S3. The phylogenetic trees of archaea. The numbers above each split were local support values. Figure S4. Mantel correlogram between the phylogenetic distances of pairwise OTUs and their niche distance of archaea.

\section{Abbreviations}

PCR: Polymerase chain reaction; rRNA: Ribosomal RNA; OTU: Operational taxonomic units; Ob: The observed number of operational taxonomic units; MNTD: Mean Nearest phylogenetic taxon distance; NTI: Nearest taxon index; PCoA: Principal coordinate analysis; dbRDA: Distance-based redundancy analysis; ANOVA: One-way analysis of variance; ANOSIM: An analysis of similarities; PERMANOVA: Permutational multivariate analysis of variance; LEfSe: Linear discriminant analysis effect size; LDA: Linear discriminant analysis; AOA: Ammonia-oxidizing archaea; SOM: Soil organic matter; TN: Total nitrogen; AN: Alkali-hydrolysable Nitrogen; $\mathrm{NH}_{4}{ }^{+}-\mathrm{N}$ : Ammonium nitrogen; TP: Total phosphorus; AP: Available phosphorus

\section{Acknowledgements}

Not applicable.

\section{Authors' contributions}

ZM conceived and designed the study. ZM, CL and HM performed the experiments. ZM, JW and GJ collected and provided the soil samples. ZM and $\mathrm{HY}$ reviewed and edited the manuscript. All authors read and approved the manuscript.

\section{Funding}

This work was financially supported by the National Key Research and Development Program of China (2017YFC0506804) and the State Key Joint Laboratory of Environmental Simulation and Pollution Control (18Y01ESTCT).

\section{Availability of data and materials}

The raw archaeal sequences in this study were deposited in Sequence Read Archive (SRA) of NCBI database and were available under accession number SRP193081.

\section{Ethics approval and consent to participate}

Not applicable.

\section{Consent for publication}

Not applicable.

\section{Competing interests}

The authors have no conflicts of interest with any parties or individuals.

Received: 5 June 2020 Accepted: 19 July 2020

Published online: 01 August 2020

\section{References}

1. Prashar P, Kapoor N, Sachdeva S. Rhizosphere: its structure, bacterial diversity and significance. Rev Environ Sci Bio. 2013;13:63-77.

2. Bulgarelli D, Garrido-Oter R, Münch PC, Weiman A, Dröge J, Pan Y, McHardy AC, Schulze-Lefert P. Structure and function of the bacterial root microbiota in wild and domesticated barley. Cell Host Microbe. 2015;17:392-403.

3. Philippot L, Raaijmakers JM, Lemanceau P, van der Putten WH. Going back to the roots: the microbial ecology of the rhizosphere. Nat Rev Microbiol. 2013;11:789-99.

4. Alegria Terrazas R, Giles C, Paterson E, Robertson-Albertyn S, Cesco S, Mimmo T, Pii Y, Bulgarelli D. Plant-microbiota interactions as a driver of the mineral turnover in the rhizosphere. Adv Appl Microbiol. 2016;95:1-67.

5. Zhalnina K, Louie KB, Hao Z, Mansoori N, da Rocha UN, Shi S, Cho H, Karaoz U, Loqué D, Bowen BP, Firestone MK, Northen TR, Brodie EL. Dynamic root exudate chemistry and microbial substrate preferences drive patterns in rhizosphere microbial community assembly. Nat Microbiol. 2018;3:470-80.

6. Yang J, Kloepper JW, Ryu CM. Rhizosphere bacteria help plants tolerate abiotic stress. Trends Plant Sci. 2009;14:1-4.
7. Castrillo G, Teixeira PJ, Paredes SH, Law TF, de Lorenzo L, Feltcher ME, Finkel OM, Breakfield NW, Mieczkowski P, Jones CD, Paz-Ares J, Dangl JL. Root microbiota drive direct integration of phosphate stress and immunity. Nature. 2015:543:513-8.

8. Lu T, Ke M, Lavoie M, Jin Y, Fan X, Zhang Z, Fu Z, Sun L, Gillings M, Peñuelas J, Qian H, Zhu YG. Rhizosphere microorganisms can influence the timing of plant flowering. Microbiome. 2018;6:231.

9. Peiffer JA, Aymé S, Omry K, Zhao J, Susannah Green T, Dangl JL, Buckler ES, Ley RE. Diversity and heritability of the maize rhizosphere microbiome under field conditions. P Natl Acad Sci USA. 2013;110:6548-53.

10. Chaparro JM, Badri DV, Vivanco JM. Rhizosphere microbiome assemblage is affected by plant development. ISME J. 2014;8(4):790-803.

11. Reinhold-Hurek B, Bünger W, Burbano CS, Sabale M, Hurek T. Roots shaping their microbiome: global hotspots for microbial activity. Annu Rev Phytopathol. 2015;53:403-24.

12. Moissl-Eichinger C, Pausan M, Taffner J, Berg G, Bang C, Schmitz RA. Archaea are interactive components of complex microbiomes. Trends Microbiol. 2018;26:70-85.

13. Spang A, Saw JH, Jørgensen SL, Zaremba-Niedzwiedzka K, Martijn J, Lind AE, van Eijk R, Schleper C, Guy L, Ettema TJG. Complex archaea that bridge the gap between prokaryotes and eukaryotes. Nature. 2015; 521:173-9.

14. Ma M, Du HX, Sun T, An SW, Yang G, Wang DY. Characteristics of archaea and bacteria in rice rhizosphere along a mercury gradient. Sci Total Environ. 2019;650:1640-51.

15. Liang B, Zhang K, Wang LY, Liu JF, Yang SZ, Gu JD, Mu BZ. Different diversity and distribution of archaeal community in the aqueous and oil phases of production pluid from high-temperature petroleum reservoirs. Front Microbiol. 2018;9:841.

16. Huang $M$, Chai $L$, Jiang $D$, Zhang $M$, Zhao $Y$, Huang $Y$. Increasing aridity affects soil archaeal communities by mediating soil niches in semi-arid regions. Sci Total Environ. 2019;647:699-707.

17. Kleikemper J, Pombo SA, Schroth MH, Sigler WV, Pesaro M, Zeyer J. Activity and diversity of methanogens in a petroleum hydrocarbon-contaminated aquifer. Appl Environ Microb. 2005;71(1):149-58.

18. Wang MZ, Wang SY, Long X, Zhuang LJ, Zhao X, Jia ZJ, Zhu GB. High contribution of ammonia-oxidizing archaea (AOA) to ammonia oxidation related to a potential active $A O A$ species in various arable land soils. J Soils Sediments. 2019;19:1077-87.

19. Khelifi N, Amin Ali $\mathrm{O}$, Roche $\mathrm{P}$, Grossi $\mathrm{V}$, Brochier-Armanet $\mathrm{C}$, Valette $\mathrm{O}$, Ollivier B, Dolla A, Hirschler-Réa A. Anaerobic oxidation of long-chain nalkanes by the hyperthermophilic sulfate-reducing archaeon, Archaeoglobus fulgidus. ISME J. 2014;8(11):2153-66.

20. Sorokin DY, Messina E, Smedile F, Roman P, Jss D, Ciordia S, Mena MC, Ferrer M, Golyshin PN, Kublanov IV, Samarov NI, Toshchakov SV, La Cono V, Yakimov MM. Discovery of anaerobic lithoheterotrophic haloarchaea, ubiquitous in hypersaline habitats. ISME J. 2017;11:1245-60.

21. Yarwood SA, Baldwin AH, Mateu MG, Buyer JS. Archaeal rhizosphere communities differ between the native and invasive lineages of the wetland plant Phragmites australis (common reed) in a Chesapeake Bay subestuary. Biol Invasions. 2016;18:1-12

22. Zhang J, Zhou X, Chen L, Chen Z, Chu J, Li Y. Comparison of the abundance and community structure of ammonia oxidizing prokaryotes in rice rhizosphere under three different irrigation cultivation modes. World J Microb Biot. 2016;32:85.

23. Chen XP, Zhu YG, Xia Y, Shen JP, He JZ. Ammonia-oxidizing archaea: important players in paddy rhizosphere soil? Environ Microbiol. 2008;10: 1978-87.

24. Lu Y, Fu L, Lu Y, Hugenholtz F, Ma K. Effect of temperature on the structure and activity of a methanogenic archaeal community during rice straw decomposition. Soil Biol Biochem. 2015;81:17-27.

25. He Y, Hu W, Ma D, Lan H, Yang Y, Gao Y. Abundance and diversity of ammonia-oxidizing archaea and bacteria in the rhizosphere soil of three plants in the Ebinur Lake wetland. Can J Microbiol. 2017;63(7):573-82.

26. Nemergut DR, Schmidt SK, Fukami T, O'Neill SP, Bilinski TM, Stanish LF, Knelman JE, Darcy JL, Lynch RC, Wickey P, Ferrenberg S. Patterns and processes of microbial community assembly. Microbiol Mol Biol R. 2013;77: 342-56.

27. Zhang B, Zhang J, Liu Y, Shi P, Wei G. Co-occurrence patterns of soybean rhizosphere microbiome at a continental scale. Soil Biol Biochem. 2018;118:178-86. 
28. Woese CR, Kandler O, Wheelis ML. Towards a natural system of organisms: proposal for the domains Archaea, Bacteria, and Eucarya. P Natl Acad Sci USA. 1990:87:4576-9.

29. Yan MM, Chen SN, Huang TL, Li BQ, Li N, Liu KW, Zong RR, Miao YT, Huang $X$. Community compositions of phytoplankton and eukaryotes during the mixing periods of a drinking water reservoir: dynamics and interactions. Int J Environ Res Public Health. 2020;17(4):1128.

30. Wen J, Zhang JQ, Nie ZL, Zhong Y, Sun H. Evolutionary diversifications of plants on the Qinghai-Tibetan plateau. Front Genet. 2014;5:4.

31. Wang L, Abbott RJ, Zheng W, Chen P, Wang Y, Liu J. History and evolution of alpine plants endemic to the Qinghai-Tibetan plateau: Aconitum gymnandrum (Ranunculaceae). Mol Ecol. 2010;18:709-21.

32. Shi $Y$, Adams JM, Ni Y, Yang $T$, Jing $X$, Chen $L$, He JS, Chu $H$. The biogeography of soil archaeal communities on the eastern Tibetan plateau. Sci Rep-UK. 2016:6:38893.

33. Zhang Y, Dong S, Gao Q, Liu S, Zhou H, Ganjurjav H, Wang X. Climate change and human activities altered the diversity and composition of soil microbial community in alpine grasslands of the Qinghai-Tibetan plateau. Sci Total Environ. 2016;562:353-63.

34. Yuan YL, Si GC, Wang J, Luo TX, Zhang GX. Bacterial community in alpine grasslands along an altitudinal gradient on the Tibetan plateau. FEMS Microbiol Ecol. 2014;87:121-32.

35. Wang JT, Cao P, Hu HW, Li J, Han LL, Zhang LM, Zheng YM, He JZ. Altitudinal distribution patterns of soil bacterial and archaeal communities along Mt. Shegyla on the Tibetan plateau. Microb Ecol. 2015;69:135-45.

36. Guo GX, Kong WD, Liu JB, Zhao JX, Du HD, Zhang XZ, Xia PH. Diversity and distribution of autotrophic microbial commu- nity along environmental gradients in grassland soils on the Tibetan plateau. Appl Microbiol Biotechnol. 2015;99:8765-76.

37. Stegen JC, Lin X, Konopka AE, Fredrickson JK. Stochastic and deterministic assembly processes in subsurface microbial communities. ISME J. 2012;6: 1653-64.

38. Edwards J, Johnson C, Santos-Medellin C, Lurie E, Podishetty NK, Bhatnaga S, Eisen JA, Sundaresan V. Structure, variation, and assembly of the rootassociated microbiomes of rice. P Natl Acad Sci USA. 2015;112:911-20.

39. Hannula SE, Morrien E, de Hollander M, van der Putten WH, van Veen JA, de Boer W. Shifts in rhizosphere fungal community during secondary succession following abandonment from agriculture. ISME J. 2017;11:2294-304.

40. Yan Y, Kuramae EE, de Hollander M, Klinkhamer PGL, van Veen JA. Functional traits dominate the diversity-related selection of bacterial communities in the rhizosphere. ISME J. 2017;11:56-66.

41. Zhang K, Adams JM, Shi Y, Yang T, Sun R, He D, Ni Y, Chu Y. Environment and geographic distance differ in relative importance for determining fungal community of rhizosphere and bulk soil. Environ Microbiol. 2017; 19(9):3649-59.

42. Jones DL, Nguyen C, Finlay RD. Carbon flow in the rhizosphere: carbon trading at the soil-root interface. Plant Soil. 2009;321:5-33.

43. Huang XF, Chaparro JM, Reardon KF, Zhang R, Shen Q, Vivanco JM. Rhizosphere interactions: root exudates, microbes, and microbial communities. Botany. 2014;92:267-75

44. Koranda M, Schnecker J, Kaiser C, Fuchslueger L, Kitzler B, Stange CF, Sessitsch A, Zechmeister-Boltenstern S, Richter A. Microbial processes and community composition in the rhizosphere of european beech-the influence of plant C exudates. Soil Biol Biochem. 2011;43:551-8.

45. Haichar FZ, Marol C, Berge O, Rangel-Castro JI, Prosser JI, Balesdent J, Heulin T, Achouak W. Plant host habitat and root exudates shape soil bacterial community structure. ISME J. 2008;2:1221-30.

46. Hinsinger $P$, Bengough AG, Vetterlein D, Young IM. Rhizosphere: biophysics, biogeochemistry and ecological relevance. Plant Soil. 2009:321:117-52.

47. LiU Y, Li H, Liu QF, Li YH. Archaeal communities associated with roots of the common reed (Phragmites australis) in Beijing Cuihu wetland. World J Microb Biot. 2015;31:823-32.

48. Brochier-Armanet C, Boussau B, Gribaldo S, Forterre P. Mesophilic crenarchaeota: proposal for a third archaeal phylum, the Thaumarchaeota. Nat Rev Microbiol. 2008;6:245-52.

49. Wu Y, Ke X, Hernandez M, Dumont MG, Jia Z, Conrad R. Autotrophic growth of bacterial and archaeal ammonia oxidizers in freshwater sediment microcosms incubated at different temperatures. Appl Environ Micro. 2013; 79:3076-84.

50. Dong K, Kim WS, Tripathi BM, Adams J. Generalized soil Thaumarchaeota community in weathering rock and saprolite. Microb Ecol. 2015;69:356-60.
51. Auguet JC, Triadó-Margarit X, Nomokonova N, Camarero L, Casamayor EO Vertical segregation and phylogenetic characterization of ammoniaoxidizing Archaea in a deep oligotrophic lake. ISME J. 2012;6:1786-97.

52. Müller $\mathrm{O}$, Wilson B, Paulsen ML, Rumińska A, Armo HR, Bratbak G, Øvreås L. Spatiotemporal dynamics of ammonia-oxidizing Thaumarchaeota in distinct arctic water masses. Front Microbiol. 2018;9:24.

53. Norman JS, Barrett JE. Substrate availability drives spatial patterns in richness of ammonia-oxidizing bacteria and archaea in temperate forest soils. Soil Biol Biochem. 2016;94:169-72.

54. Galloway JN, Dentener FJ, Capone DG, Boyer EW, Howarth RW, Seitzinger SP, Asner GP, Cleveland C, Green P, Holland E. Nitrogen cycles: past, present, and future. Biogeochemistry. 2004;70:153-226.

55. Wang Y, Li C, Kou Y, Wang J, Tu B, Li H, Li X, Wang C, Yao M. Soil pH is a major driver of soil diazotrophic community assembly in QinghaiTibet alpine meadows. Soil Biol Biochem. 2017;115:547-55.

56. Shen C, Xiong J, Zhang H, Feng Y, Lin X, Li X, Liang W, Chu H. Soil pH drives the spatial distribution of bacterial communities along elevation on Changbai Mountain. Soil Biol Biochem. 2013;57:204-11.

57. Zhang T, Wang NF, Liu HY, Zhang YQ, Yu LY. Soil pH is a key determinant of soil fungal community composition in the Ny-Ålesund region, Svalbard (high Arctic). Front Microbiol. 2016;7:227.

58. Fan K, Weisenhorn P, Gilbert JA, Shi Y, Bai Y, Chu H. Soil pH correlates with the co-occurrence and assemblage process of diazotrophic communities in rhizosphere and bulk soils of wheat fields. Soil Biol Biochem. 2018;121:185-92.

59. Hu HW, Zhang LM, Yuan CL, He JZ. Contrasting Euryarchaeota communities between upland and paddy soils exhibited similar pH-impacted biogeographic patterns. Soil Biol Biochem. 2013;64:18-27.

60. Yuan $\mathrm{CL}$, Zhang LM, Wang JT, Hu HW, Shen JP, Cao P, He JZ. Distributions and environmental drivers of archaea and bacteria in paddy soils. J Soils Sediments. 2018;19:23-37.

61. Gubry-Rangin C, Hai B, Quince C, Engel M, Thomson BC, James P, Schloter M, Griffiths RI, Prosser JI, Nicola GW. Niche specialization of terrestrial archaeal ammonia oxidizers. P Natl Acad Sci USA. 2011;108:21206-11.

62. Lei L, Gundersen P, Tao Z, Mo J. Effects of phosphorus addition on soil microbial biomass and community composition in three forest types in tropical China. Soil Biol Biochem. 2012;44:31-8.

63. Horner-Devine MC, Bohannan BJ. Phylogenetic clustering and overdispersion in bacterial communities. Ecology. 2006;87:100-8.

64. Feng M, Adams JM, Fan K, Shi Y, Sun R, Wang D, Guo X, Chu H. Long-term fertilization influences community assembly processes of soil diazotrophs. Soil Biol Biochem. 2018;126:151-8.

65. Chesson P. Mechanisms of maintenance of species diversity. Annu Rev Eco Syst. 2000;31:343-66.

66. Violle C, Nemergut DR, Pu Z, Jiang L. Phylogenetic limiting similarity and competitive exclusion. Ecol Lett. 2011;14:782-7.

67. Peng C, Jiang H, Huang L, Yang J, Wang S, Huang Q, Deng S, Dong $\mathrm{H}$. Abundance and diversity of ammonia-oxidizing bacteria and archaea in cold springs on the Qinghai-Tibet plateau. Geomicrobiol J. 2013;30:530-9.

68. Wu X, Xu H, Liu G, Ma X, Mu C, Lin Z. Bacterial communities in the upper soil layers in the permafrost regions on the Qinghai-Tibetan plateau. Appl Soil Ecol. 2017;120:81-8.

69. Dini-Andreote F, Stegen JC, van Elsas JD, Salles JF. Disentangling mechanisms that mediate the balance between stochastic and deterministic processes in microbial succession. P Natl Acad Sci USA. 2015;112(11):1326-32.

70. Langenheder S, Székely AJ. Species sorting and neutral processes are both important during the initial assembly of bacterial communities. ISME J. 2011 5:1086-94.

71. Zhou J, Deng Y, Zhang P, Xue K, Liang Y, Van Nostrand JD, et al. Stochasticity, succession, and environmental perturbations in a fluidic ecosystem. Proc Natl Acad Sci U S A. 2014;111:E836-45.

72. Shi S, Nuccio EE, Shi ZJ, He Z, Zhou J, Firestone MK. The interconnected rhizosphere: high network complexity dominates rhizosphere assemblages. Ecol Lett. 2016;19:926-36.

73. Mendes LW, Kuramae EE, Navarrete AA, van Veen JA, Tsai SM. Taxonomical and functional microbial community selection in soybean rhizosphere. ISME J. 2014:8:1577-87.

74. Berry D, Widder S. Deciphering microbial interactions and detecting keystone species with co-occurrence networks. Front Microbiol. 2014;5:219.

75. Harrison KA, Bol R, Bardgett RD. Do plant species with different growth strategies vary in their ability to compete with soil microbes for chemical forms of nitrogen. Soil Biol Biochem. 2008;40:228-37. 
76. Chen J, Carrillo Y, Pendall E, Dijkstra FA, Dave Evans R, Morgan JA, Williams DG. Soil microbes compete strongly with plants for soil inorganic and amino acid nitrogen in a semiarid grassland exposed to elevated $\mathrm{CO}_{2}$ and warming. Ecosystems. 2015;18:867-80.

77. Watts DJ, Strogatz SH. Collective dynamics of 'small-world' networks. Nature. 1998:393:440-2.

78. Zhou J, Deng Y, Luo F, He Z, Tu Q, Zhi X. Functional molecular ecological networks. MBio. 2010;1:1592-601.

79. Berry $\mathrm{D}$, Widder S. Deciphering microbiali interactions and detecting keystone species with co-occurrence networks. Front Microbiol. 2014;5:219.

80. Zhou YJ, Li JH, Ross Friedman C, Wang HF. Variation of soil bacterial communities in a chronosequence of rubber tree (Hevea brasiliensis) plantations. Front Plant Sci. 2017;8:849.

81. Yang B, Xu H, Wang J, Song X, Wang Y, Li F, Tian Q, Ma C, Wang D, Bai J, Sand W. Bacterial and archaeal community distribution and stabilization of anaerobic sludge in a strengthen circulation anaerobic (SCA) reactor for municipal wastewater treatment. Bioresour Technol. 2017;244:750-8.

82. Caporaso JG, Kuczynski J, Stombaugh J, Bittinger K, Bushman FD, Costello EK, et al. QIIME allows analysis of high-throughput community sequencing data. Nat Methods. 2010;7:335-6.

83. Aronesty E. Comparison of sequencing utility programs. Open Bioinformatics I. 2013;7:1-8.

84. Kembel SW, Cowan PD, Helmus MR, Cornwell WK, Morlon H, Ackerly DD Blomberg SP, Webb CO. Picante: R tools for integrating phylogenies and ecology. Bioinformatics. 2010;26:1463-4.

85. Wang J, Shen J, Wu Y, Tu C, Soininen J, Stegen JC, He J, Liu X, Zhang L, Zhang E. Phylogenetic beta diversity in bacterial assemblages across ecosystems: deterministic versus stochastic processes. ISME J. 2013;7: 1310-21.

86. Dumbrell AJ, Nelson M, Helgason T, Dytham C, Fitter AH. Relative roles of niche and neutral processes in structuring a soil microbial community. ISME J. 2010:4:337-45.

87. Langfelder $\mathrm{P}$, Horvath S. Fast R functions for robust correlations and hierarchical clustering. J Sta Softw. 2012;46(11):i11.

88. Bastian M, Heymann S, Jacomy M. Gephi: an open source software for exploring and manipulating networks. International AAAI Conference on Weblogs and Social Media. 2009;8:361-2.

\section{Publisher's Note}

Springer Nature remains neutral with regard to jurisdictional claims in published maps and institutional affiliations.

Ready to submit your research? Choose BMC and benefit from:

- fast, convenient online submission

- thorough peer review by experienced researchers in your field

- rapid publication on acceptance

- support for research data, including large and complex data types

- gold Open Access which fosters wider collaboration and increased citations

- maximum visibility for your research: over $100 \mathrm{M}$ website views per year

At $\mathrm{BMC}$, research is always in progress.

Learn more biomedcentral.com/submissions 